\title{
Role of Structured Feedback of Direct Observation of Procedural Skills in improving Clinical Skill of Interns
}

Georgy J Eralil

\section{ABSTRACT}

Objectives:

- To assess clinical skills of interns in selected procedures using direct observation of procedural skills (DOPS)

- To assess the change in procedural skills among students who received a structured feedback on DOPS.

Materials and methods: The study was interventional and conducted between December 2015 and February 2016 at the Department of Obstetrics and Gynaecology of Sree Narayana Institute of Medical Sciences, Ernakulam, Kerala, India. In the study, investigator observed the trainee performing a practical procedure within the workplace and recorded a rating for each competency on the assessment form. Feedback was given expanding on the reasons for any ratings of development required and makes practical suggestions for any remedial steps. The intern is reassessed for the same procedure at a later stage and the impact on skills is scored. Then the structured feedback is provided, and later on again the score on skills obtained by DOPS are compared between pre- and post-values by Wilcoxon-signed rank test.

Results: The average of pre- and post-feedback values were compared by Wilcoxon signed rank test. The p-value calculated was $<0.001$, which is significant. None of the interns were competent to perform the procedure before feedback; $80 \%$ of them needed more practice and $20 \%$ needed supervision. After feedback, $53.3 \%$ achieved competency and $46.7 \%$ achieved competency needing supervision; $93.3 \%$ of interns regarded feedback as superior quality, while $6.7 \%$ regarded as satisfactory.

Conclusion: Direct observation of procedural skills can be used to assess clinical skills of interns in selected procedures and it brings changes in procedural skills among students who received a structured feedback on DOPS.

Keywords: Direct observation of procedural skills, Feedback, Internship, Medical education, Skill assessment, Workplacebased assessment.

How to cite this article: Eralil GJ. Role of Structured Feedback of Direct Observation of Procedural Skills in improving Clinical Skill of Interns. J Postgrad Med Edu Res 2017;51(1):22-27.

Associate Professor

Department of Obstetrics and Gynecology, Sree Narayana Institute of Medical Sciences, Ernakulam, Kerala, India

Corresponding Author: Georgy J Eralil, Associate Professor Department of Obstetrics and Gynecology, Sree Narayana Institute of Medical Sciences, Ernakulam, Kerala, India, Phone: +914842624589, e-mail: georgyeralil@yahoo.co.in
Source of support: Harsha $\mathrm{CH}$, Statistician, Sree Narayana Institute of Medical Sciences, Ernakulam, Kerala, India

Conflict of interest: None

\section{INTRODUCTION}

Assessment is a concept which we are all familiar with, as each stage of a medical career is marked by the successful completion of examinations. Assessments are likely to play an increasing role in continuing professional development and revalidation in the future, meaning that clinicians are likely to be assessed more regularly and also required to take on the assessor role more frequently. Effective, valid, and reliable approaches for assessment are therefore required to measure knowledge, skills, and attitudes.

With fast and serious changes of assessing methods in medical education, new approaches with optimal serious impact have been developed, mostly focusing on clinical proficiencies. ${ }^{1}$ One of these methods is direct observation of procedural skills (DOPS). In this method, subjects are observed in an environment quite similar to the actual activities, in which there is a real patient and procedure and they focus on important points of the considered skill required to be evaluated. ${ }^{2}$ This method makes it easier to provide feedback to students, and contributes to a feedback given based on actual and perceived behaviors instead of general comments. Therefore, it is not only considered as motivation and learning encouragement for students but also gives direction to their learning efforts and indicates principal matters, regarding the direct relevance of test style and content with clinical performance. ${ }^{3}$ It shows how to achieve the desired goals and skills, and motivates learners trying to improve and enhance clinical practice. ${ }^{4,5}$ It is noteworthy that in order to maximize training impact of this method, the subjects' strength and weakness points should be identified. Feedbacks need to be provided with high sensitivity and in a suitable environment immediately after the assessment, and the students' weaknesses be emphasized in addition to the strengths. ${ }^{3}$

Direct observation of procedural skills is one of a number of assessments used in the clinical setting to help the teaching and assessment of a clinical skill in the workplace. In common with the other workplace-based 
assessments (WBAs) ${ }^{6,7}$ Its primary purpose is to provide structured teaching and feedback in a particular area of clinical practice. It is applicable to short, diagnostic, and interventional procedures, or part procedures, that comprise relatively few steps. It will be found most useful during the early years of surgical training.

Patient safety and well-being remain paramount throughout. The assessor supervising the procedure should ensure that the patient is informed, has provided consent for the exercise, and suffers no increased risk or discomfort. The supervisor retains responsibility for patient care throughout and will intervene as the situation requires.

\section{OBJECTIVES}

- To assess clinical skills of interns in selected procedures

- To assess the change in procedural skills among students who received a structured feedback on DOPS.

\section{MATERIALS AND METHODS}

\section{Study Design}

Interventional

\section{Study Population}

The study population includes interns in Department of Obstetrics and Gynecology.

\section{Study Period}

December 2015 to February 2016.

\section{Sample Size}

Sixty observations (30 pre-feedback and 30 post-feedback) by the investigator, the interns may be recurring.

\section{Site}

Department of Obstetrics and Gynecology, Sree Narayana Institute of Medical Sciences, Ernakulam, Kerala, India.

\section{Investigator}

Georgy Joy Eralil, Associate Professor, Obstetrics and Gynecology, Sree Narayana Institute of Medical Sciences, Ernakulam, Kerala, India.

The assessment involved an assessor observing the trainee performing a practical procedure within the workplace. The procedures tested were venepuncture, suturing of episiotomy, closure of skin incision, cervical smear, and urinary catheterization. The assessor's evaluation was recorded on a printed version of the structured assessment form which was used by the assessor to provide the trainee with structured feedback. The assessor observed the trainee undertaking the procedure and doing what they would normally do in that situation. Most procedures took no longer than 15 to 20 minutes. Assessor recorded a rating for each competency on the assessment form. ${ }^{8,9}$ The assessment form was modified including the feedback of intern and was peer-reviewed.

The assessment settings were Accident and Emergency, Operation Theater, Outpatients, and Labor Ward. It must be emphasized that the most important purpose of the assessment exercise was to provide the trainee with formative feedback, offering a significant impact on learning. Scores were used only for the purpose of identifying strengths and weaknesses and providing accurate feedback on that performance. Each item was scored D (development required or needs improvement or satisfactory only after help or advice), or S (satisfactory standard for completion of early years with no prompts required).

Feedback took about 5 to 10 minutes. It was conducted in a suitable, quiet environment immediately after the assessment and was constructive expanding on the reasons for any ratings of development required and made practical suggestions for any remedial steps.

It was essential that trainees reflect on feedback and took a proactive approach to improving their practice. Each $D$ carries 0 and $S$ carries +1 . The intern was reassessed for the same procedure at a later stage and the impact on skills was scored. First objective was achieved by describing the number of students who scored D grade/S grade initially. Then the structured feedback was provided and later on again the skills are scored by DOPS. Compare pre- and post-values by Wilcoxon-signed rank test (nonparametric test for paired variables).

The assessor checked whether intern demonstrated knowledge by clearly explaining to the assessor the indication for the procedure, the relevant anatomy, and essential steps of the procedure.

The assessor checked the professional behavior throughout the procedure like obtaining consent, after explaining procedure and possible complications to patient, communicating clearly with patient and staff throughout the procedure. I checked whether intern conveys information, i.e., complete, relevant, clear and jargon free, sensitive to patient's concerns, respects confidentiality, actively listens, answers questions correctly, and checks patient understanding before obtaining consent and establishes trust. I also checked whether intern demonstrates respect, and understanding of the patient's needs for comfort, respect, and confidentiality; 
demonstrates an ethical approach, awareness of any relevant legal frameworks; makes a postoperative assessment; conveys relevant information orally and in writing; and retains responsibility for the patient's ongoing care.

Checked whether intern prepares for procedure according to an agreed protocol by demonstrating clear preoperative plan, identifying any special equipment required, making all relevant safety checks, briefing other staff appropriately to select appropriate local anesthetic agent (or sedative) and checks with nursing staff; injects appropriate volume using the correct needle and technique; performs the technical aspects in line with the guidance notes following the protocol for the procedure; demonstrates good technique; uses instruments appropriately; handles tissue gently; controls bleeding appropriately; sutures skin neatly and traumatically. The assessor checked whether intern demonstrates good asepsis and safe use of instruments and sharps by following high standards of aseptic operative technique; handles instruments and sharps safely. The assessor checked whether intern deals with any unexpected event or seeks help when appropriate anticipates and responds calmly and appropriately, communicates clearly and consistently with patients and staff, uses assistants to best advantage, has awareness of own limitations.

Post procedure management includes safe disposal of instruments and completes required documentation by making clear and legible notes that enable effective care by other practitioners. The assessor checked whether intern labels samples gave clear instructions of monitoring and arranged after care.

\section{DATA ANALYSIS}

The mean for pre-feedback score was 2.5 and the postfeedback score was 6.4. The median for pre-feedback score was 2 and post-feedback score was 6.5. The average values

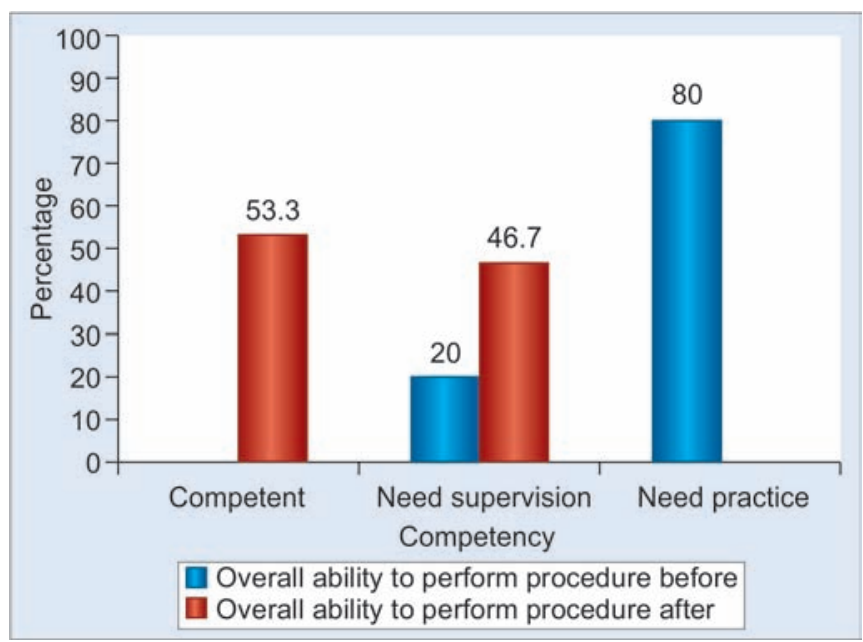

Graph 1: Overall ability to perform before feedback compared to after feedback of pre-feedback and post-feedback values are compared by Wilcoxon-signed rank test. The p-value calculated was $<0.001$, i.e., significant (Table 1).

None of the interns were competent to perform procedure before feedback. About $80 \%$ of them needed more practice and $20 \%$ needed supervision. After feedback, $53.3 \%$ achieved competency and $46.7 \%$ achieved competency needing supervision. Graph 1 shows 93.3\% of interns regarded feedback as superior quality, while Graph 2 shows $6.7 \%$ regarded as satisfactory.

\section{DISCUSSION}

The study shows that there is significant improvement in procedural skills of interns after receiving a structured feedback. Deconstruction of operations into their component parts enables trainees to practice on simple simulations representing each component, and be assessed as competent, before undertaking the actual operation. Assessment of surgical competence by direct observation is feasible and reliable; such assessments could be used for both formative and summative assessment. ${ }^{10}$ Procedure-based assessment is a reliable and acceptable method of assessing surgical skills, with good construct validity. Whatever WBA method is used, the purpose, timing, and frequency of assessment require detailed guidance.

The limitations were smaller number, time allotted to study was limited. Further research is required into

Table 1: Descriptive statistics

\begin{tabular}{|c|c|c|c|c|c|}
\hline & Mean & Median & $\begin{array}{l}\text { Standard } \\
\text { deviation }\end{array}$ & Range & $p$-value \\
\hline $\begin{array}{l}\text { Pre-feedback } \\
\text { score }\end{array}$ & 2.5 & 2 & 1.137 & 5 & $<0.001$ \\
\hline $\begin{array}{l}\text { Post-feedback } \\
\text { score }\end{array}$ & 6.4 & 6.5 & 0.67 & 2 & \\
\hline
\end{tabular}

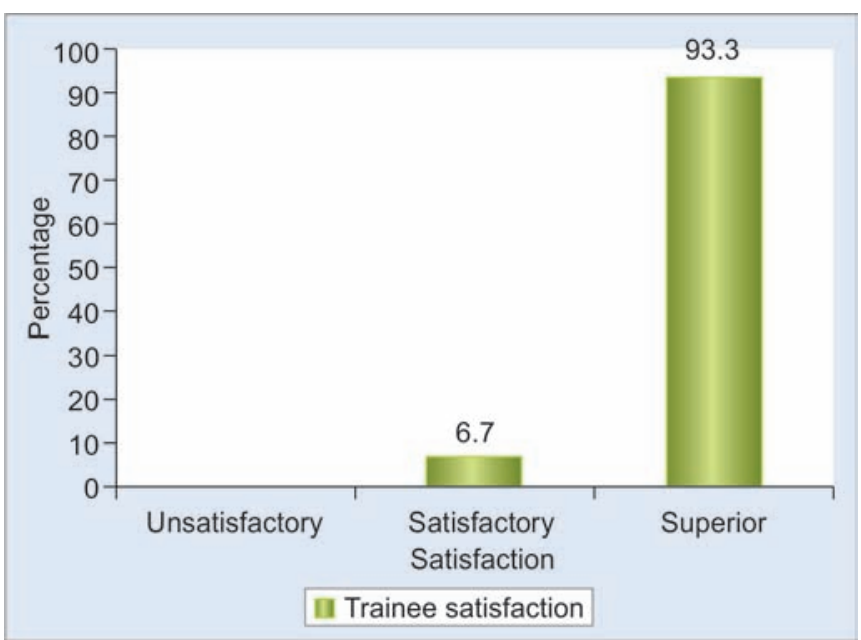

Graph 2: Trainee satisfaction 
the use of health care professionals to assess trainees, the relationship between performance and experience, the educational impact of assessment, and the additional value of video recording. ${ }^{11}$

The average time taken for feedback was 10 minutes. The WBA not only provides the opportunity to observe and assess in the real-life situation but also to provide a feedback for improvement at the most appropriate time. The utility of assessments is theorized as a product of validity, reliability, feasibility, acceptability, and educational influence. In practice, there may be several tradeoffs between these key elements in various assessments with repercussions. Whilst an assessment may be designed to have an overall utility, it may score poorly on certain aspects.

Inherently, WBA tools score well on construct validity, by virtue of being set in real clinical situations at the workplace. It provides for observation of a wide variety of clinical work in an authentic setting. The reliability of WBA is often debated but it is also argued that, since most WBA tools involve many encounters with a number of assessors spread over a period of time, their reliability may be considered acceptable with six to eight encounters per tool per year. However, there are significant feasibility issues relating to achieving this frequency of encounters.

The landmark meta-analysis by Hattie established the importance of feedback as an important contributor to learning. ${ }^{12}$ Feedback is most effective when given for specific tasks. Despite clear evidence in support, the power of observation of actual clinical work and feedback remains grossly underutilized in medical education. Majority of interns in study considered feedback given was of superior quality. While not many such data is available in India, studies from Western countries suggest that less than one-third of clinical encounters are actually observed during training. ${ }^{13,14}$ At the postgraduate level, up to $80 \%$ of postgraduate students may have only one observed clinical encounter. ${ }^{15}$ The above facts make it amply clear that not only there is a limitation in terms of number of opportunities available for direct observation and feedback but also gross underutilizations of these sparse opportunities.

Direct observation of procedural skills is a highly structured tool, which is most applicable in assessing the mechanistic technicalities of procedural skills. A structured form of evaluation is preferable to other crude measures of assessment as structured evaluations result in outcomes that are more reliable and the assessments are more effective. ${ }^{16}$ In some training programs, structural form of evaluation is replacing other more crude measures of procedural competence with poor validity and reliability, such as logbooks and supervisor evaluations. ${ }^{17}$ Several studies have found a lack of rigorous testing of procedural skills. ${ }^{17}$ To address this deficiency, DOPS is designed to assess the procedural skills of surgical, medical, or general practice trainees at all levels. A drawback of DOPS is that it evaluates a specific encounter, which may not be representative of a trainees overall performance, rather than rating based on assessment over a longer period of time and that specific encounter. ${ }^{18}$ The consequential validity of WBA is much debated in the literature; it has been suggested that the dynamic nature of clinical work is poorly served by the quantitative performance data and psychometric focus inherent in WBA. Several concerns arise about the trainee manipulating the assessment process, such as altering their behavior depending on their assessor and regarding the WBA as a mini high-stake examination, thereby putting pressure on the assessor to award a "pass."

The acceptability and utility of WBA hinges on the sensitization of assessors and trainees, the cultivation of an educational learning environment, and the training of assessors in providing constructive feedback. These are all adjustable dynamics that can be improved with deliberate effort in the correct educational pathway.

\section{CONCLUSION}

In medical education, WBA is now a fixture. A clear identification of encounters as being either formative or summative is to be welcomed, particularly in enabling the key educational objective of achieving documented, quality feedback. The validity of the WBA is more robust when the purpose of encounters is clearly identified and understood in this way by both trainer and trainee. The re-engagement of both the trainer and trainee facilitated by a move away from a tick-box exercise is to be encouraged, as the educational value of assessment will benefit. The provision of formal feedback from a senior trainer and reflective practice from the trainee is the major benefit of WBAs; with care and the right culture, the apprentice model of training may be reestablished and the full educational potential of WBAs can be realized. ${ }^{19}$

Hence, it can be concluded that DOPS can be used to assess clinical skills of interns in selected procedures and it brings changes in procedural skills among students who received a structured feedback on DOPS.

\section{LIMITATIONS}

- Small sample studied

- Short time of study

\section{RECOMMENDATIONS}

Further research is required into the use of health care professionals to assess trainees, the relationship between performance and experience, video recording of the procedure, and feedback and the educational impact of assessment. 
APPENDIX 1: Feedback Form

\begin{tabular}{|l|l|l|l|l|l|l|l|l|l|l|l|l|l|l|l|l|l|l|l|l|}
\hline Trainee's Forename: & & & & & & & & & & & & & & & & & & & \\
\hline Trainee's Surname: & & & & & & & & & & & & & & & & & \\
\hline Trainee's MCl: & & & & & & \multicolumn{2}{|l|}{ Date of Assessment (dd/mm/yyy): $/ 20$} \\
\hline
\end{tabular}

Procedure:

\begin{tabular}{|l|c|c|c|}
\hline Domain \& Comments & Satisfactory & Needs Improvement & Comments \\
\hline $\begin{array}{l}\text { Professional Approach (to include communication, } \\
\text { consent and consideration of the patient.) }\end{array}$ & $\square$ & $\square$ & \\
\hline Knowledge (indication, anatomy, technique). & $\square$ & $\square$ & \\
\hline $\begin{array}{l}\text { Demonstrate appropriate preparation pre- } \\
\text { procedure }\end{array}$ & $\square$ & $\square$ & \\
\hline Appropriate analgesia & $\square$ & $\square$ & \\
\hline Technical Ability & $\square$ & $\square$ & \\
\hline Aseptic Technique & $\square$ & $\square$ & \\
\hline Post Procedure Management & $\square$ & $\square$ & \\
\hline
\end{tabular}

\begin{tabular}{|c|c|c|c|}
\hline $\begin{array}{l}\text { Overall Ability to } \\
\text { perform Procedure }\end{array}$ & $\begin{array}{c}\text { Competent to } \\
\text { perform unsupervised } \\
\square\end{array}$ & $\begin{array}{c}\text { May need supervision } \\
\text { if complications arise } \\
\square\end{array}$ & $\begin{array}{c}\text { Needs more practice } \\
\square\end{array}$ \\
\hline & & & \\
\hline
\end{tabular}

Time taken for Feedback \& Observation (in minutes)

\begin{tabular}{|l|l|l|l|l|l|l|l|l|l|l|l|l|l|l|l|l|l|l|l|l|l|}
\hline Assessors Surname: & E & R & A & L & I & L & & & & & & & & & & & & & & & \\
\hline $\begin{array}{l}\text { Assessors } \\
\text { Registration No. }\end{array}$ & 3 & 1 & 2 & 5 & 6 & Assessors Signature: & \multicolumn{1}{|l|}{} \\
\hline
\end{tabular}

\begin{tabular}{|l|l|l|l|l|l|l|l|l|l|l|}
\hline \multirow{2}{*}{$\begin{array}{l}\text { Trainee } \\
\text { satisfaction }\end{array}$} & \multicolumn{4}{|c|}{ unsatisfactory } & \multicolumn{3}{c|}{ satisfactory } & \multicolumn{5}{c|}{ superior } \\
\cline { 2 - 12 } & 1 & 2 & 3 & 4 & 5 & 6 & 7 & 8 & 9 \\
\hline
\end{tabular}

\section{FEEDBACK FORM}




\section{REFERENCES}

1. Norcini JJ, McKinley DW. Assessment methods in medical education. Teach Teach Educ 2007 Apr;23(3):239-250.

2. Shahgheibi S, Pooladi A, Bahramrezaie M, Farhadifar F, Khatibi R. Evaluation of the effect of direct observation of procedural skill (DOPS) on clinical externship students learning level in obstetrics ward of Kurdistan University of Medical Sciences. J Med Educ 2009 Winter-Spring;13(1-2):29-33.

3. Jalili M. DOPS or direct observation of procedural skills [cited 2010 Jul 11]. Available from: http://jouybari.blogfa.com/ post/4472.

4. Chambers M. Some issues in the assessment of clinical practice: a review of the literature. J Clin Nurs 1998 May;7(3): 201-208.

5. Milligan F. Defining and assessing competence: the distraction of outcomes and the importance of educational process. Nurse Educ Today 1998 May;18(4):273-280.

6. Beard J, Strachan A, Davies H, Patterson F, Stark P, Ball S, Taylor P, Thomas S. Developing an education and assessment frame work for the foundation programme. Med Educ 2005 Aug;39(8):841-851.

7. Norcini J, Burch V. Workplace-based assessment as an educational tool: AMEE guide No. 31. Med Teach 2007 Nov;29(9): 855-871.

8. Kundra S, Singh T. Feasibility and acceptability of direct observation of procedural skills to improve procedural skills. Indian Paediatr 2014 Jan;51(1):59-60.

9. NHS UK etft.co.uk mandatory for GMC.

10. Beard JD, Jolly BC, Newble DI, Thomas WE, Donnelly J, Southgate LJ. Assessing the technical skills of surgical trainees. Br J Surg 2005 Jun;92(6):778-782.
11. Beard JD, Marriott J, Purdie H, Crossley J. Assessing the surgical skills of trainees in the operating theatre: a prospective observational study of the methodology. Health Technol Assess 2011 Jan;15(1):1-162.

12. Hattie JA. Influences on student learning. Inaugural professorial address [cited 1999 Aug 2]. Available from: http:/ / www. education.auckland.ac.nz/webdav/site/education/shared/ hattie/docs/influences-on-student-learning.pdf

13. Daelmans HE, Hoogenboom RJ, Donker AJ, Scherpbier AJ, Stehouwer CD, van der Vleuten CPM. Effectiveness of clinical rotations as a learning environment for achieving competences. Med Teach 2004 Jun;26(4):305-312.

14. Kogan JR, Hauer KE. Brief report: use of the mini-clinical evaluation exercise in internal medicine core clerkships. J Gen Intern Med 2006 May;21(5):501-502.

15. Day SC, Grosso LG, Norcini JJ, Blank LL, Swanson DB, Horne MH. Residents' perceptions of evaluation procedures used by their training program. J Gen Intern Med 1990 SepOct;5(5):421-426.

16. Khan MAA, Gorman M, Gwozdziewicz L, Sobani ZA, Gibson C. Direct observation of procedural skills as an assessment tool for surgical trainees. J Pak Med Stud 2013 Jul-Sep;3(3): 137-140.

17. Sidhu RS, Grober ED, Musselman LJ, Reznick RK. Assessing competency in surgery: where to begin? Surgery 2004 Jan;135(1):6-20.

18. Cipher. Review of work-based assessment methods. Sydney: Centre for Innovation in Professional Health Education \& Research; 2007.

19. Parry-Smith W, Mahmud A, Landau A, Hayes K. Workplacebased assessment: a new approach to existing tools. Obstet Gynaecol 2014 Oct;16(4):281-285. 